\title{
Design, Fabrication, and Testing of Silicon Microgimbals for Super-Compact Rigid Disk Drives
}

\author{
Viktoria Temesvary, Shuyun Wu, Wen H. Hsieh, Yu-Chong Tai, and Denny K. Miu
}

\begin{abstract}
This paper documents results related to design optimization, fabrication process refinement, and micron-level static/dynamic testing of silicon micromachined microgimbals that have applications in super-compact computer disk drives as well as many other engineering applications of microstructures and microactuators requiring significant out-of-plane motions. The objective of the optimization effort is to increase the in-plane to out-of-plane stiffness ratio in order to maximize compliance and servo bandwidth and to increase the displacement to strain ratio to maximize the shock resistance of the microgimbals, while that of the process modification effort is to simplify in order to reduce manufacturing cost. The testing effort is to characterize both the static and dynamic performance using precision instrumentation in order to compare various prototype designs. [93]
\end{abstract}

\section{INTRODUCTION}

$\mathbf{M}$ UCH HAS BEEN said about the tremendous progress made in the 1980's and the impact that it has had on our lives in terms of electronics and computers. In the coming decade, consumer electronics and personal computers will merge, propelling us into the exciting era of integrated information electronics. By combining the functions of televisions, telephones, and computers, a new generation of productivityoriented informatic electronics products will be introduced, providing us with tremendous capabilities for multimedia telecommunication and telecomputation.

In order to keep up with the promised performance, we must continue to provide much of the high-bandwidth, servocontrolled precision mechanisms and mechanical systems in order to acquire, store, distribute, and process personal and business information at previously unimaginable speed, volume and form-factor. Specifically, high-performance microelectromechanical components and systems (MEMS) must be designed and commercially manufactured in order to facilitate the introduction of a new generation of super-compact computer peripheral devices, for example, sub-2-inch-diameter magnetic recording rigid disk drives to accommodate the enormous data storage requirements in extremely challenging

Manuscript received November 26, 1993; revised November 20, 1994. This work was supported by the University of California MICRO Program and matching funds from Applied Magnetics, Hewlett-Packard, IBM, Maxtor, Quantum, and Seagate Technology.

V. Temesvary and D. Miu are wth the Mechanical Engineering Department, University of California at Los Angeles, Los Angeles, CA 90024-1597 USA.

S. Wu, W. H. Hsieh, and Y.-C. Tai are with the Department of Electrical Engineering, California Institute of Technology, Pasadena, CA 91125 USA.

IEEE Log Number 9409007.

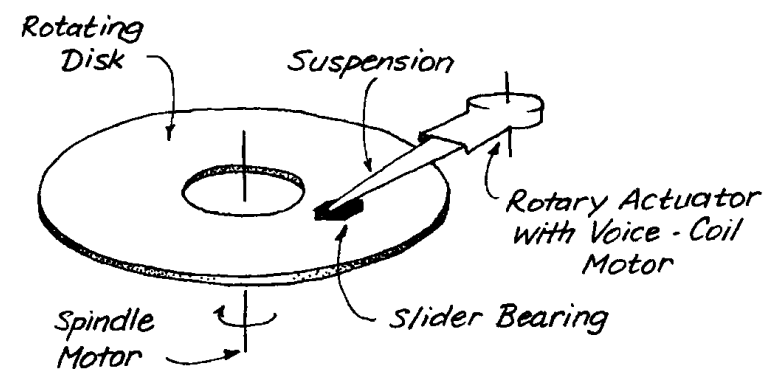

Fig. 1. Major electromechanical components of a conventional highperformance magnetic recording rigid disk drive.

and nontraditional computing environments, such as those in handheld consumer-oriented information and communication systems [6].

In two recent papers by Miu et al., we have introduced a design concept and demonstrated a preliminary fabrication process of a silicon-micromachined microgimbal for mounting sub-miniaturized $(30 \%)$ read-write heads/pico-sliders in small form factor disk drives. In the current work, we shall document additional results related to the design optimization, fabrication process refinement, and micron-level testing (both static and dynamic) of some working prototypes.

In typical high-performance rigid disk drives (Fig. 1), the major electromechanical components consist of a rotating disk coated with thin-film magnetic media, a spindle bearing/brushless DC motor, a hydrodynamic gas-lubricated slider bearing (for holding the read/write head at hundreds or even tens of nanometer spacing to the recording surface), and an electromagnetic voice-coil rotary actuator for moving the slider across the disk surface.

In addition, there also exists a stainless-steel suspension arm for attaching the slider to the actuator. In order to maintain proper head/media compliance (to maximize bit density), the design of the gimbal/suspension must be such that it is extremely soft in the vertical, pitch, and roll directions. On the other hand, in order to maximize the servo bandwidth (to increase track density), the suspension must be stiff in the remaining in-plane directions. Furthermore, it must also carry the electrical signals to and from the read/write transducers [5].

Fig. 2 shows our proposed silicon-micromachined microgimbal/suspension, which has a number of potential operational advantages. First of all, since silicon is a brittle 


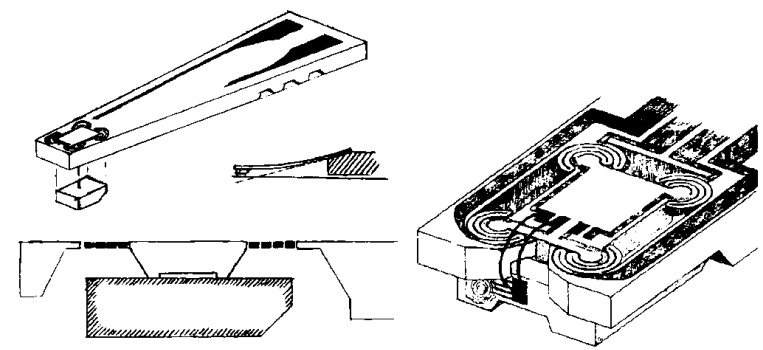

Fig. 2. The proposed design concept of an integrated silicon microgim$\mathrm{bal} / \mathrm{suspension}$ with built-in electrodes.

material, silicon suspensions cannot be plastically deformed during manufacturing and handling; quality assurance therefore consists of only visual inspection. In comparison, plastic deformation of stainless steel suspensions (i.e., de-gramming) is the major contributing factor of reduced production yield and in-drive performance problems, representing a significant cause of revenue loss.

In addition, due to the difference in process technology, much smaller features can be realized in silicon than in stainless steel (using various anisotropic wet and dry etching techniques), providing much needed additional degrees of design freedom. For example, in our design, we fabricate very intricate serpentine planar microsprings at the four corners of the microgimbal (see Fig. 3) in order to satisfy the conflicting out-of-plane compliance, lateral stiffness, and shock resistance design requirements.

Furthermore, with silicon, it is relatively easy to incorporate electrodes (or even pre-amps) in the suspension arm as well as an electrical coupler in the gimbal area, thereby allowing the possibility of automating not only the mechanical but also the electrical interconnect manufacturing procedures. Currently, in many state-of-the-art products, assembly of the head/gimbal/suspension subsystem represents nearly half of the total manual labor cost.

\section{Design Optimization}

As was described in Miu et al. [8], the design objectives of the silicon microgimbal are basically two-fold: it must have a very high in-plane/out-of-plane stiffness ratio (to satisfy both tracking bandwidth and head/media compliance requirements) while maintaining a low strain-to-displacement ratio (to maximize shock resistance). Intuitively, this corresponds to designing a planar serpentine spring with very high effective length and relatively high width-to-thickness aspect ratio. Our constraint of course is the available real estate; in order to be consistent with the product requirement, we need to maintain a specific form factor for the overall gimbal/suspension assembly which prevents us from having excessively long and wide beams.

During our initial testing, we observed that for a given out-of-plane displacement of the center coupon, the resulting deformation is not equally shared among the various concentric coils of the serpentine gimbal spring, frequently with fracture occurring at the outermost turn. It therefore seemed

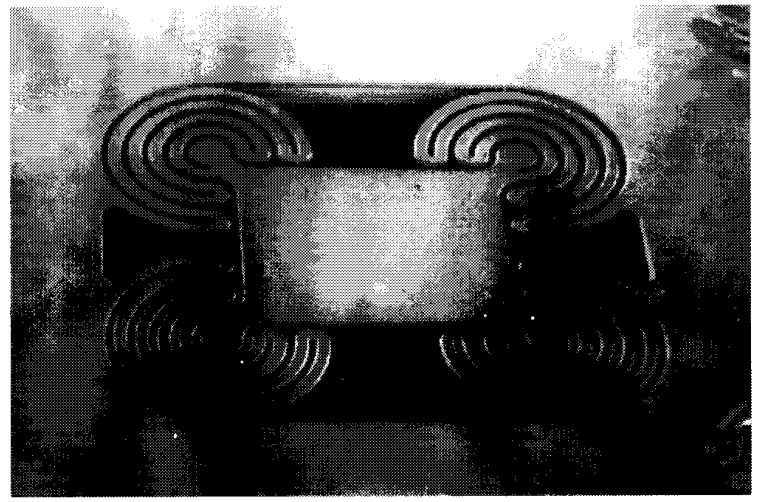

Fig. 3. SEM micrograph of a silicon microgimbal with serpentine planar microsprings at the four corners (width of the beam is about $80 \mu \mathrm{m}$ ).

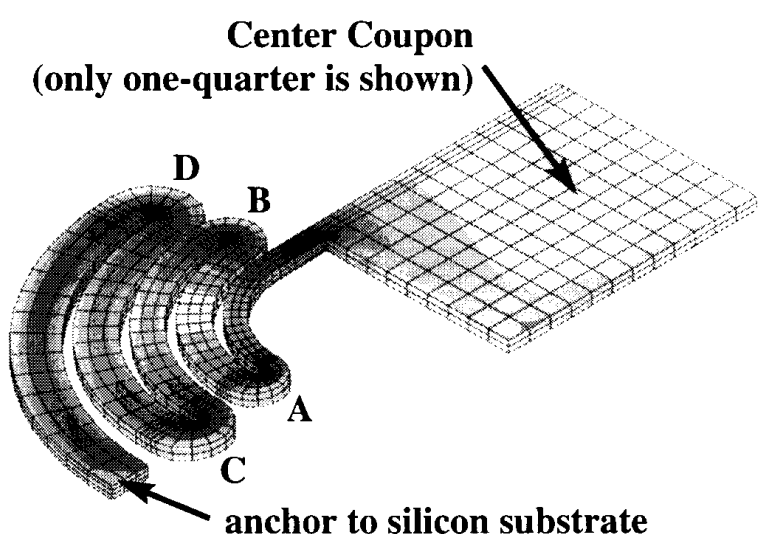

Fig. 4. Finite-element model of a silicon microgimbal with serpentine planar microsprings at the four corners (only one-quarter is shown).

logical that our first task should be to optimize the widths of the individual beams, making it wider at the outside coils than the inside, while maintaining a constant overall size.

Fig. 4 shows a finite-element model (FEM) analyzing the serpentine microgimbal as illustrated in Fig. 3, except that the current design has non-uniform beam widths and the wrap angle has been reduced from $270^{\circ}$ to $90^{\circ}$. This change in wrap angle is primarily a result of our desire to minimize the overall profile of the silicon microgimbal.

In the original design concept illustrated in Fig. 2, in order to minimize the lateral dimensions of the microgimbal, we designed the center coupon to be smaller than the slider. However, in doing so, we increased the vertical thickness of the suspension/gimbal/slider assembly since we must have enough clearance between the serpentine microsprings and the slider in order to avoid physical contact due to steady-state or transient pitching and rolling motions. For our applications, a high-profile gimbal is not acceptable since disk-to-disk spacing is currently a major performance constraint for super-compact form factor disk drives, which would impede any effort to maintain the traditional volumetric data density advantage of magnetic storage products over solid-state memory devices and optical storage systems. 


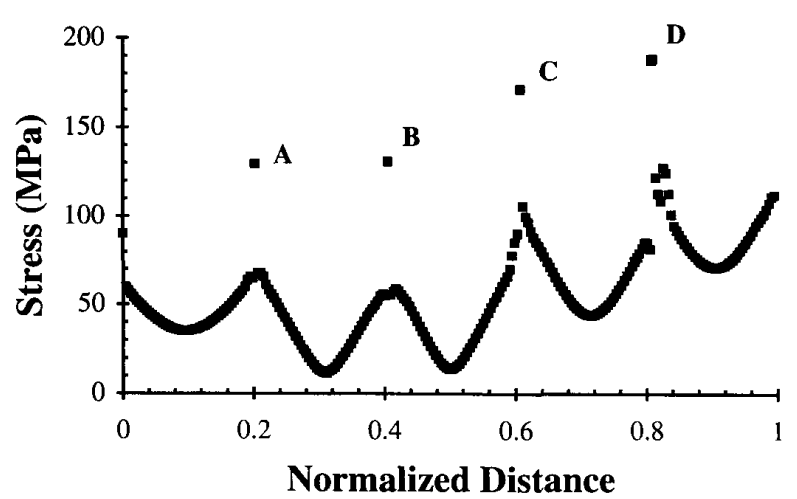

Fig. 5. Von Mises stress along the length of the serpentine microgimbal (with constant widths)

In the latest design, we have relocated the serpentine microsprings outside of the slider and instead of putting them symmetrically at the four corners as is shown in Figs. 2 and 3, we put them in the front and back of the center coupon (which now has the same size as the slider), enabling us to have zero clearance between the center coupon and the slider, hence a lower-profile microgimbal. As a result, the wrap angle of the individual serpentine microspring is reduced to $90^{\circ}$, which has the additional benefit of a higher in-plane/out-of-plane stiffness ratio [8].

Fig. 5 shows FEM results analyzing a serpentine microspring design with uniform widths, where the Von Mises stresses of each element along the center-line of the beam are plotted for the entire length of the beam, starting at the anchor point at the center coupon and ending at the outside where the gimbal spring is attached to the silicon substrate. Since at the vicinity of the various $180^{\circ}$ turns (points A through D), the stress states are quite complicated (see Fig. 4), we have summarized the results by plotting only the peak stresses around the turns.

From Fig. 5, one can observe that the serpentine spring is basically a collection of five individual curved cantilever beams joined together end-to-end, each with boundary conditions similar to those of clamped-clamped beams such that the stresses are always higher at the turns (i.e., clamped ends) and lower at the mid-spans. Furthermore, as expected the stresses are higher at the outside coil with the critical point being the very last turn (point $\mathrm{D}$ ). To better utilize the available real estate, we proceed to optimize the widths of the individual beams, such as by lowering the peak stresses and making them more uniformly distributed.

In order to properly compare the results from various FEM runs, however, the calculated stresses need to be normalized since in addition to stresses, the spring constants will also change as a function of beam geometry. In practice, we typically wish to achieve a particular spring stiffness as required by the applications. So as we change the width, we need to compensate by varying the beam thickness in order to keep the stiffness and therefore the loading conditions constant.

For design purposes, instead of iterating the beam thickness $t$, it is better to normalize the calculated stress $\sigma$ with stiffness

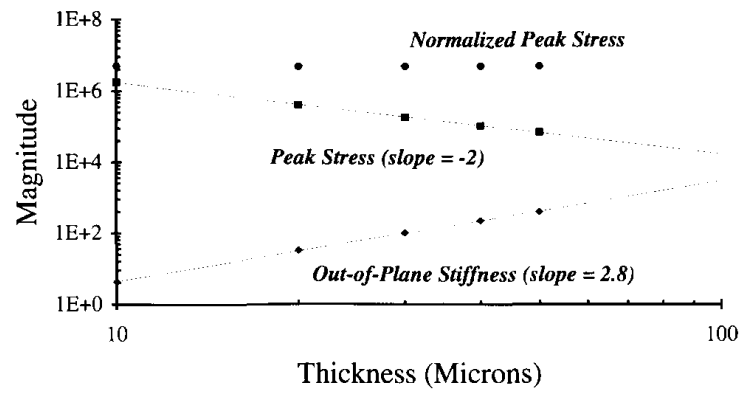

Fig. 6. Magnitudes of calculated peak stress, normalized peak stress, and out-of-plane stiffness as a function of gimbal thickness.

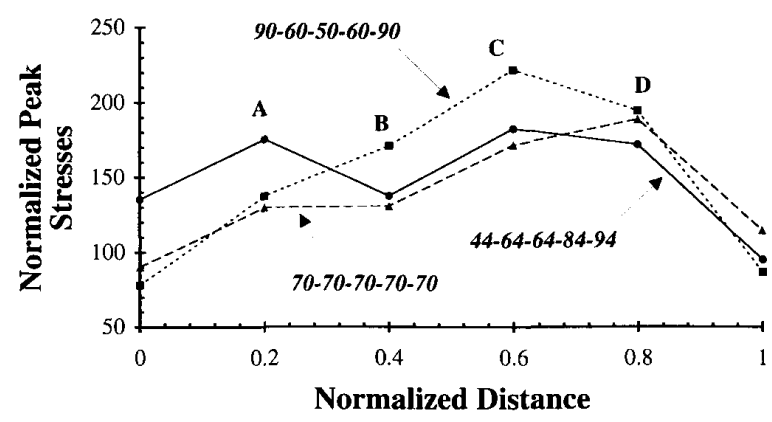

Fig. 7. Comparison of normalized peak stresses for serpentine microgimbals with different beam widths.

$K$ such that we can compare quantities that are independent of $t$. Recall that for a simple cantilever beam, $K$ varies with $t^{3}$ (from FEM results, we actually have $K \propto t^{2.8}$ for the more complicated serpentine spring as shown in the bottom trace of Fig. 6) while $\sigma$ varies with $t^{-2}$ (middle trace). Consequently, to be independent of $t$, we have multiplied all calculated values of $\sigma$ by $K^{2 / 3}$ (or more accurately, by $K^{2 / 2.8}$ as shown in the top trace of Fig. 6).

Fig. 7 compares the normalized peak stresses of three serpentine gimbal designs with various beam widths. The one annotated with long-dashes has a constant width of $70 \mu \mathrm{m}$ and is the same as that shown in Fig. 5. When the width of the inner most coil is reduced to $44 \mu \mathrm{m}$ (as shown in the trace with solid line), the peak stress of the inside turn (point A) goes up as expected. However, with the excess real estate, we can afford to increase the widths of the two outer coils (to 84 and $94 \mu \mathrm{m}$, respectively), which has the net effect of bringing down the peak stress of the overall design (point $\mathrm{D}$ ). Therefore, this is a much better design than the one with uniform widths, confirming our intuition.

On the other hand, if the widths of both the inner and outer most coils are increased (to $90 \mu \mathrm{m}$ as is done with the trace annotated with short-dashes), then it is possible for stresses at the middle turn (point $\mathrm{C}$ ) to significantly increase beyond that of the last turn (point D), moving the critical point inward and as a result, making it a weaker design. In our prototype serpentine gimbal design, we have therefore adopted nonuniform widths, increasing progressively from the inside to the outside. 


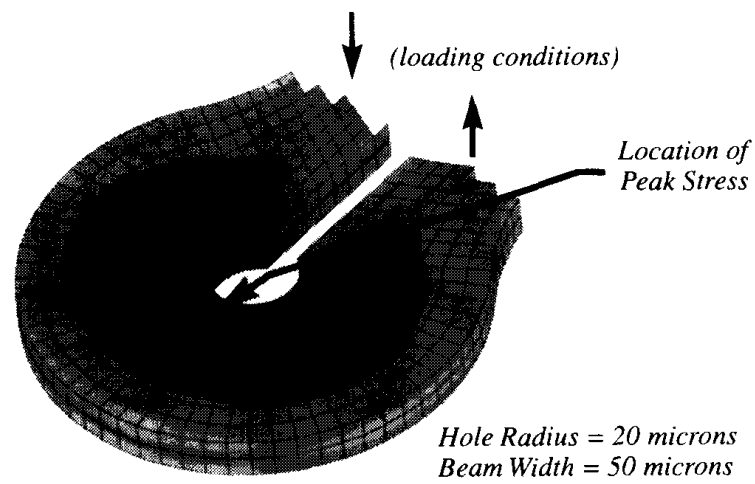

Fig. 8. Finite-element model of the serpentine microspring with a stress relief hole.

It should be noted that besides the first-order effect of reducing the peak stress, varying the widths of the serpentine springs also changes other second-order performance parameters, such as in-plane stiffness and resonant frequencies. A much more elaborate optimization scheme than the one described here would have to be used in order to more accurately quantify the various design tradeoffs.

Our second optimization task has to do with reducing the stress concentration around the various $180^{\circ}$ turns, which have been the primary failure points. Fig. 8 shows a detailed FEM analyzing the stresses around a single turn where we have fixed the beam width to $50 \mu \mathrm{m}$, spacing between beams to $20 \mu \mathrm{m}$, and outside radius to $100 \mu \mathrm{m}$.

Fig. 9 shows the calculated peak stress around the turn and the corresponding out-of-plane stiffness as functions of the inside radius of the stress relief hole, which varies from 0 (sharp corners) to $70 \mu \mathrm{m}$. It is quite clear that as the stress relief hole gets bigger, the spring will become softer since there is less structural material to support the load. On the other hand, the peak stress is quite high when there is no relief hole (radius $=0$ ) and decreases substantially when a circular hole is introduced (giving the sharp turn some curvature). This is so even when the actual load-carrying area has been reduced. However, it is clear that when the radius of the hole increases beyond a critical dimension, the disadvantage of a narrower beam far outweights the advantage of the stress relief hole and the peak stress increases.

In our prototype design, the radius of the stress relief hole is typically between 20 to $30 \mu \mathrm{m}$ (the exact dimension depends on the amount of undercut introduced during the fabrication process). For a truly optimized design, the size of the stress relief holes should vary with the beam width. But for simplicity, we have compromised on a fixed hole size here.

\section{Process IMPRovement}

The fabrication process for the silicon microgimbal is rather simple and consists of only two major steps: reactive-ion etching (RIE) using $\mathrm{SF}_{6} / \mathrm{O}_{2}$ plasma to define the microspring on the front surface of the wafer and anisotropic chemical etching on the back to create a trapezoidal cavity which would

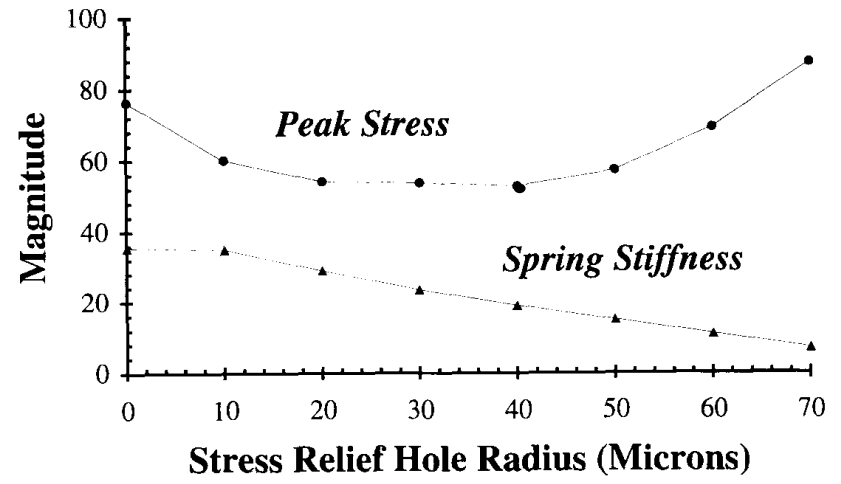

Fig. 9. Calculated peak stresses and corresponding spring stiffnesses for serpentine microsprings with stress relief holes of various radius.

allow the attachment of the read/write slider directly under the center coupon. For etch stop, we use epi-wafers which consist of a 20 - to $30-\mu$ m-thick lightly doped epitaxial layer on top of a $4-\mu \mathrm{m}$ heavily boron-doped buried layer. ${ }^{1}$

In [8], we have described the original process flow where RIE was performed first using electron-beam evaporated aluminum as the mask, followed by low-pressure chemical-vapor deposition (LPCVD) of low-temperature oxide (LTO) serving as the protection mask for subsequent chemical etching. Although not necessary at the time, we had chosen LTO instead of other high-temperature processes (such as thermal exide or LPCVD nitride) because we envisioned incorporating metal electrodes in the final products. However, LTO has the disadvantage that step coverage is usually very poor, which requires thick (about $2 \mu \mathrm{m}$ ) and therefore long (about 2 hours) deposition, making it an expensive process step.

For the anisotropic etching, we used ethylenediamine pyrocatechol (EDP), which has the advantage that it does not attack silicon oxide (as opposed to potassium hydroxide, $\mathrm{KOH}$ ), but it has the disadvantage that it is carcinogenic and has been (or will soon be) banned from usage in any industrial settings. One of our first tasks was to find a replacement for EDP.

We have tried different compositions of ammonium hydroxides, for example, ammonium hydroxide, and water, $\mathrm{NH}_{4} \mathrm{OH}$, and tetramethyl ammonium hydroxide, $\left(\mathrm{CH}_{3}\right)_{4} \mathrm{NOH}$ or TMAH [9]. After some experimentation, we finalized on TMAH because its etch rate of silicon is about the same as that of EDP and although its etch rate of silicon oxide is higher, it is tolerable (see Table I for etch rate data for TMAH and EDP). ${ }^{2}$ Also, it is commercially available and is relatively inexpensive. However, it does have a number of disadvantages compared to EDP.

First of all, the selectivity of TMAH for undoped over boron-doped silicon is much lower than that of EDP (only about $20: 1$ to $100: 1$ depending on the process temperature and doping concentration as opposed to much more than $500: 1$ for EDP), which makes the boron-layer a much less

\footnotetext{
${ }^{1}$ The doping also contains a small amount of germanium to help minimize residual stresses; see [3] for more details.

${ }^{2}$ We wish to thank our colleagues Ms. Svetlana Tatić-Lučić and Mr. Fukang Jiang for their experimental data and references on EDP etching.
} 
TABLE I

Experimentally Obtained Etch Rates of Silicon and SLLICON OXIDE FOR TMAH (15\% WT) AND EDP AT Various Process Temperatures (See Also [10], [11])

Etch rate of (100) $\mathrm{Si}, \mu \mathrm{m} /$ hour Selectivity of Si to LPCVD LTO Selectivity of undoped to bonon-deopd $\mathrm{Si}\left(2 \times 10^{20}\right.$ atoms/cc) Selectivity of undoped to bonon-deopd Si $\left(1 \times 10^{21}\right.$ atoms $/ \mathrm{cc}$ ) $92: 1$ $>500: 1$
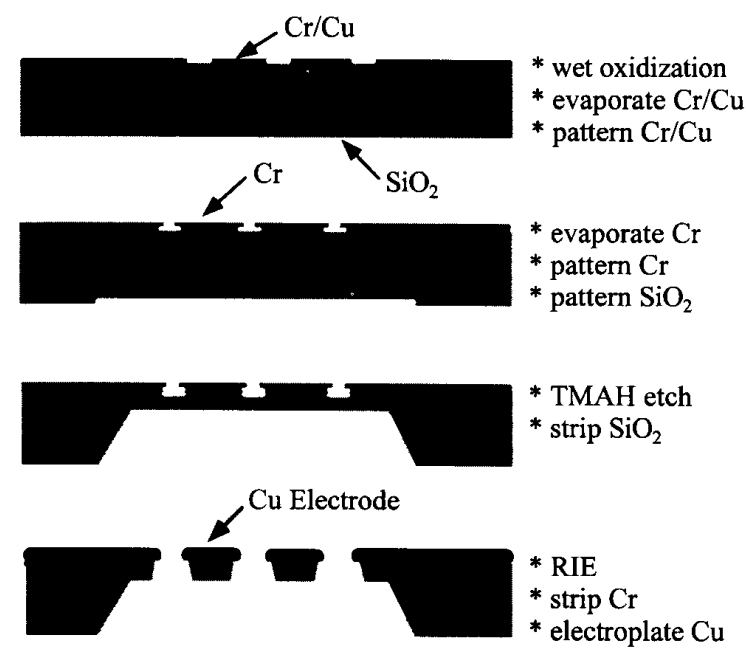

Fig. 10. Processing steps for the silicon serpentine microgimbal.

effective etch stop in TMAH. As a result, we typically etch at $90^{\circ} \mathrm{C}$ for about $7-1 / 2$ hours and then switch to $80^{\circ} \mathrm{C}$ for the remaining time with very careful monitoring.

The other problem with TMAH has to do with corner compensation. Our experience with TMAH is that the relationship between the etch rates along different crystalline planes is not a constant and varies significantly depending on the age and concentration of the chemical. This makes the design of the corner compensators an almost impossible task. In our original process, in addition to cavities (which have no protruding corners), we also etch troughs around the gimbal/suspension in order to individualize the finished parts. In our new process, we have eliminated all corners from the mask and as a result, the parts are now separated using a dicing saw. This requires some careful considerations during mask design in order to avoid sawing through any part of the wafer that has been back-etched.

Our second task in improving the original process is to eliminate LPCVD. One alternative to LPCVD oxide is thermal oxide. But in order to do thermal oxidization, we must reverse the process such that TMAH is performed before RIE. The reason is more than just simply wanting to perform hightemperature processes prior to metalization. More importantly,

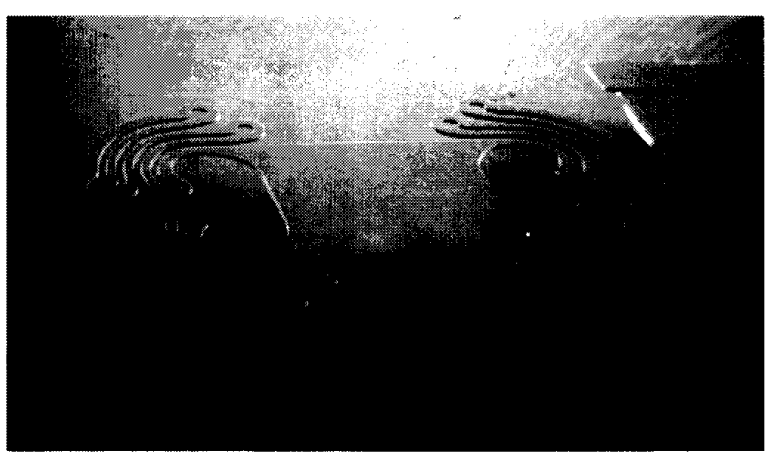

Fig. 11. SEM micrograph of a five-turn silicon microgimbal fabricated using the improved process.

once RIE is performed, the boron-layer would be exposed and during thermal oxidation, boron would diffuse into the silicon oxide (and into silicon), lowering the concentration of the etch-stop layer. Subsequent chemical etching would then cause severe undercuts in the microstructures, making them extremely fragile.

The original reason for wanting to do back-side TMAH etch at the very end is that we want to perform as many of the critical photolithography process steps as possible on a robust substrate. Therefore the only practical way of reversing the process would be to do it in such a way that all lithographic steps (including the one for the metal electrodes) are performed prior to both TMAH and RIE. Fortunately, this is possible since TMAH does not attack certain metals (such as nickel or chrome, or even aluminum if enough silicon has been dissolved in the solution).

Fig. 10 shows a summary of the improved processing steps that require only three masks. The silicon wafer is first thermally oxidized at $1050^{\circ} \mathrm{C}$ to form a $3000-\AA$-thick silicon oxide layer on the top and bottom surfaces, which serves as the protection coating for TMAH as well as insulation for the electrical interconnect. Two-hundred- $\AA$-thick chrome and $1000-\AA$ copper are then thermally evaporated on the top surface and patterned using the first mask to form a seed layer for the electrodes. A 2000 - $\AA$-thick chrome is evaporated on top of the seed layer and patterned using the second mask to define the microsprings. The third mask is used to pattern the silicon oxide on the back surface for the cavities.

Once the photolithographic steps are finished, the wafer is ready for TMAH. Since the copper seed layer is protected by the chrome RIE mask, it will not be attacked by TMAH. After TMAH back-etch and removal of silicon oxide, we perform RIE using pure $\mathrm{SF}_{6}$ until the microgimbals are completely free-standing. This is then followed by chemical removal of the chrome, once again revealing the copper seed layer. The very last step before dicing is to electroplate the copper electrodes. Fig. 11 shows an example of the microgimbals fabricated using the improved process described in Fig. 10, which was designed and optimized using the finite-element model shown in Fig. 4. All test structures documented in the remainder of this paper are fabricated using this new process (TMAH instead of EDP, etc.). 


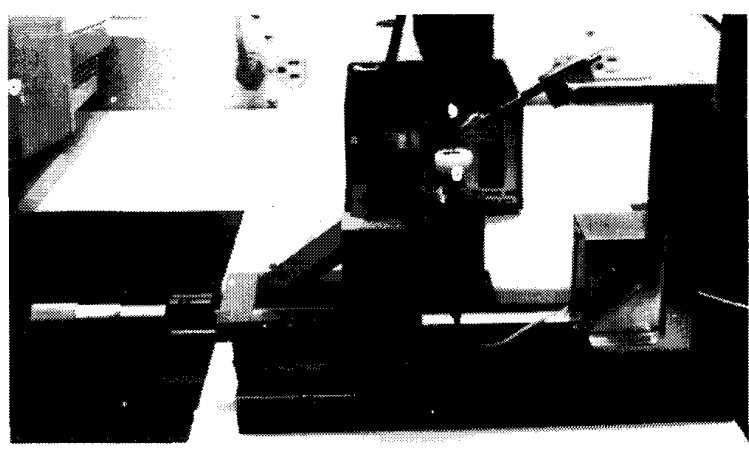

Fig. 12. Specially developed, high-precision static-load tester for silicon microgimbals.

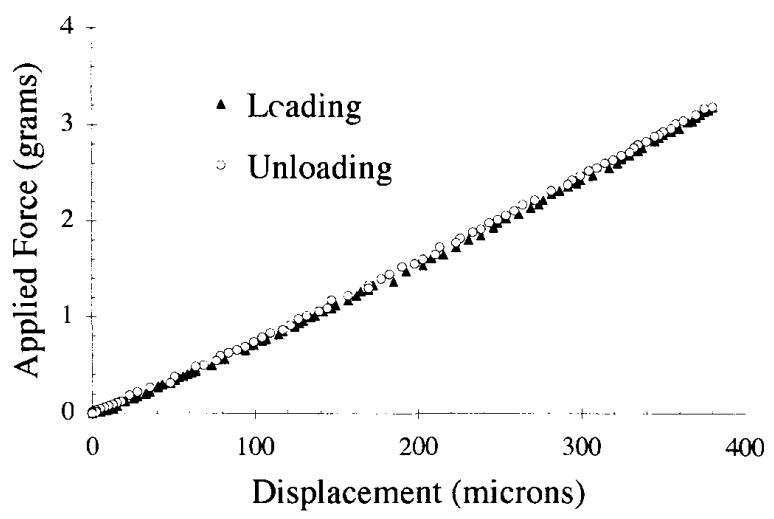

Fig. 13. Loading and unloading of a five-turn silicon serpentine microgimbal.

\section{Static/Dynamic Testing}

Testing of the silicon microgimbals involves both static and dynamic measurements. Fig. 12 shows a specially designed high-precision mechanical fixture for static testing. At the center of the picture (the small white disk) is a holder for the silicon specimen, which is mounted on top of a load-cell (the stainless steel cylinder) for measuring applied force. The load-cell (which has a 10-grams maximum range with a 0.01 grams resolution) is in turn mounted on a $x-y-z$ microstage for positioning the specimen under a fixed sharp stylus (the angled probe above the holder; see also Fig. 14 for details). In addition, on the vertical stage, there is a LVDT (linear voltage differential transformer) for measuring the vertical displacement of the microgimbal (with $2.5-\mu \mathrm{m}$ resolution). ${ }^{3}$

Fig. ' 13 shows an example of the measured data where we plot the resulting applied force (in grams) as a function of imposed vertical penetration of the probe (in microns). It can be seen that the stiffness of the microgimbal is very linear (the spring constant of this particular microgimbal is about 80 $\mathrm{N} / \mathrm{m}$ ). In addition, as expected, silicon does not exhibit any hysteresis behavior during the load-unload cycle.

In subsequent experiments, we have tested many specimens to fracture and the maximum load for the five-turn design is

${ }^{3}$ We wish to thank Drs. Paul Smith and Josh Harrison of the Applied Magnetic Corporation for their assistance in building the static-load tester.

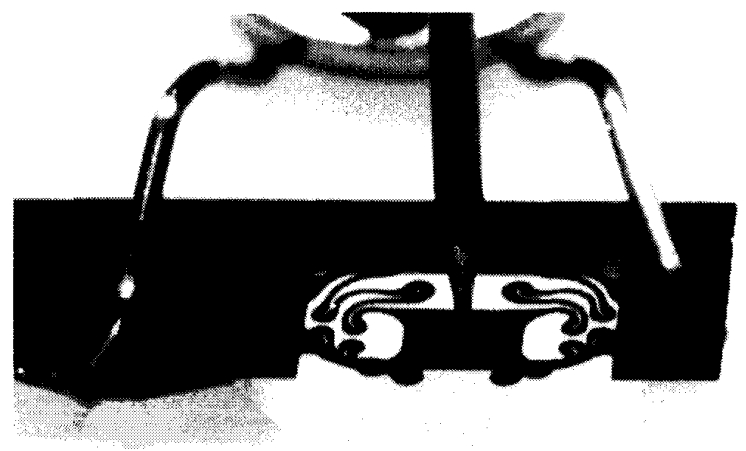

Fig. 14. Close-up view of a five-turn silicon microgimbal under static loading,

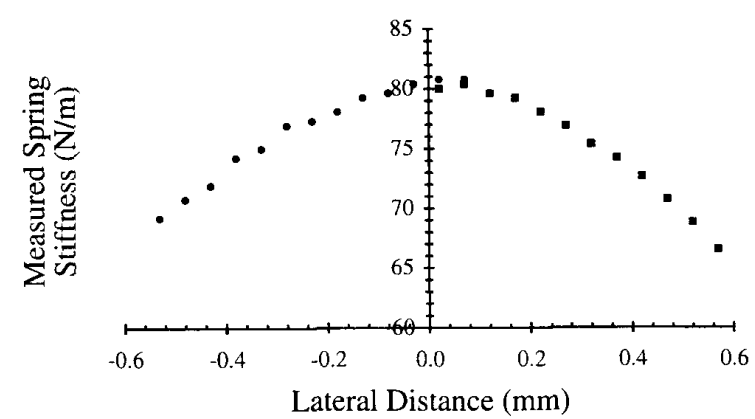

Fig. 15, Measured stiffness as a function of lateral distance for a five-turn silicon serpentine microgimbal.

typically on the order of 4 to 5 grams (corresponds to about 2000 to $2500 \mathrm{~g}$ 's of acceleration). In fact, some specimens were able to sustain maximum out-of-plane displacements on the order of $1 \mathrm{~mm}$ (for a $20-\mu \mathrm{m}$-thick microgimbal)! Fig. 14 shows a close-up view of the deformed microgimbal (similar to the one shown in Fig. 11).

For our applications, in addition to vertical stiffness, it is also very important to characterize the pitch and roll stiffness of the microgimbals. If we assume the center coupon to be rigid, then the stiffness constants are related simply as follows:

$$
\frac{1}{K_{m}}=\frac{1}{K_{z}}+\frac{r^{2}}{K_{\theta}}
$$

where $K_{z}$ and $K_{\theta}$ are the equivalent vertical and pitch stiffness, respectively, and $K_{m}$ is the actual measured stiffness when the load point is some lateral distance $r$ away from the stiffness centroid of the microgimbal. Very often the location of the centroid (where the measured stiffness is maximum, i.e., no contribution from pitch) can be slightly different from the geometric center (usually less than $50 \mu \mathrm{m}$ ) due to the nonuniformity of the finished corner serpentine microsprings. In Fig. 15, we have plotted the measured stiffness of the microgimbal as a function of lateral distance.

From Fig. 15 we can indeed observe the inverse square relationship between measured stiffness $K_{m}$ and distance $r$. On the other hand, in Fig. 16, we have re-plotted the same data in terms of compliance versus distance squared. It can be 


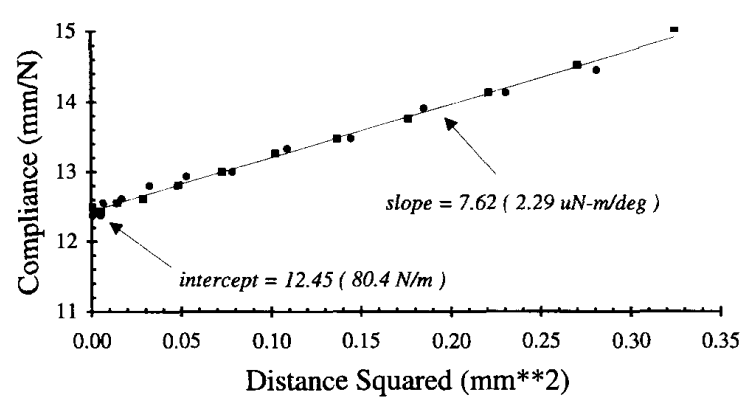

Fig. 16. Spring compliance plotted as a function of distance squared.

TABLE II

Comparison of Measured Vertical StiffNess (N/m) and Pitch aNd Roll StiffNess ( $\mu N$-m/Deg.) OF THE SILICON Microgimbals Using Static and Dynamic Measurement Techniques

\begin{tabular}{lllllll}
\hline & \multicolumn{3}{c}{ 5-turn coil } & \multicolumn{3}{c}{ 3-turn coil } \\
\cline { 2 - 7 } & vert & pitch & roll & vert & pitch & roll \\
\hline $\begin{array}{c}\text { static (w/o } \\
\text { slider) }\end{array}$ & 80 & 2.29 & 0.62 & 183 & 6.85 & 1.25 \\
$\begin{array}{c}\text { static } \\
\text { (w/slider) }\end{array}$ & 88 & 2.07 & 0.65 & 224 & 5.24 & 1.50 \\
$\begin{array}{c}\text { dynamic } \\
\text { (w/slider) }\end{array}$ & 87 & 1.86 & 0.71 & 223 & 4.91 & 1.46 \\
\hline
\end{tabular}

seen that the relationship is linear as described by (1). From Fig. 16, we can easily calculate the slope which is equal to the reciprocal of the equivalent pitch stiffness $K_{\theta}$ and the intercept which is that of the vertical stiffness $K_{z}$. Our experience is that compared to a single-point measurement, this is a more reliable method of accurately characterizing the spring stiffness of the microgimbals.

Table II summarizes the results of static measurements for two prototype microgimbals, one with five turns (the data of which have been shown in Figs. 13, 15, and 16) and a slightly different design which has only three turns. It can be seen that as expected, the stiffness of the microgimbal is much higher when there are fewer number of turns (the stiffness is about 2.3 times higher for vertical, three times for pitch and two times for roll).

We have also tested the microgimbals with a $30 \%$ slider bonded under the center coupon. ${ }^{4}$ If the coupon is rigid, then bonding of a slider will have no effect on the static stiffness. However, one can observe that there is a slight increase in the spring constant (except for pitch) suggesting that the coupon is in fact flexible. It also makes sense that the effect is higher for the stiffer microgimbal since in comparison, much more deformation will be exerted on the flexible coupon and therefore more contribution to the overall compliance (the increase in vertical stiffness is about $20 \%$ for the stiffer three-turn design but only $10 \%$ for the five-turn).

In addition to static measurement, we also performed dynamic measurements. Fig. 17 shows a laser Doppler vibrometer (LDV) setup designed especially for measuring micronlevel in-plane and out-of-plane dynamic motion of the silicon microgimbals (as well as other MEMS devices). At the center

\footnotetext{
${ }^{4}$ We wish to thank Dr. Chak Leung of the Read-Rite Corporation for his assistance in obtaining the dummy sliders.
}

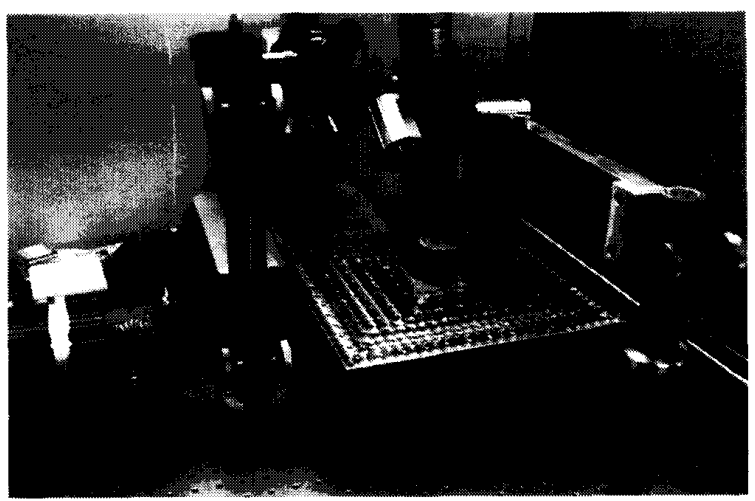

Fig. 17. Laser Doppler Vibrometer (LDV) setup for noncontact interferometric dynamic testing of microgimbals (see [1]).

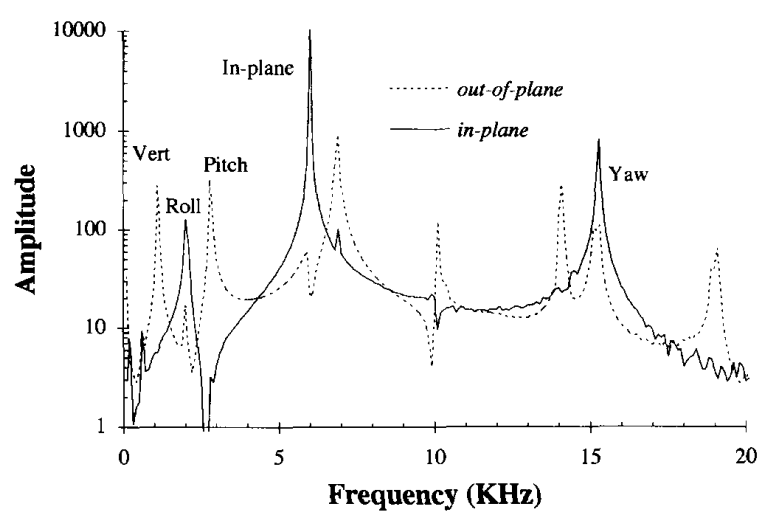

Fig. 18. Measured frequency spectra of in-plane and out-of-plane motions of a five-turn silicon microgimbal.

of Fig. 17 is a small piezoelectric shaker (the small ceramic disk on top of the polished aluminum plate) for mounting and exciting the microgimbals.

On the left hand side of Fig. 17 is the commercially available LDV which consists of a frequency stabilized HeNe laser and an acoustooptical modulator for upshifting the laser frequency by $40 \mathrm{MHz}$. The shifted signal beam emitted by the LDV is redirected and focused onto the microgimbal mounted on a high-resolution motorized $x-y$ stage, allowing very precise positioning of the specimen under microscope. The reflected beam is then collected by the LDV and recombined with the reference beam to provide a frequency-modulated (FM) signal with a carrier at $40 \mathrm{MHz}$ and a modulation signal proportional to the velocity of excited motion of the microgimbal.

This signal is then fed to a FM demodulator and the output signal is connected to a spectrum analyzer which also provides the excitation signal for the shaker. The frequency spectra captured at various locations on the microgimbal are then sent to a laboratory workstation which stores the data and composes the animation to display the various mode shapes.

Fig. 18 shows the measured spectra for both in-plane and out-of-plane motions of the five-turn silicon microgimbal (with slider attached). The three lowest out-of-plane modes correspond to the vertical, roll, and pitch motions. The first 
TABLE III

Comparison of Measured Resonant Frequencies $(\mathrm{kHz})$ aND IN-Pl.ane AND OLT-OF-PLANE MOTIONS OF THE SILICON Microgimbals with and witholt Sliders Attached

\begin{tabular}{ccccccccc}
\hline & \multicolumn{4}{c}{ 5-turn coil } & \multicolumn{4}{c}{ 3-turn coil } \\
\cline { 2 - 9 } & plane & vert & pitch & roll & plane & vert & pitch & roll \\
山WO & - & 3.8 & 6.5 & 5.8 & - & 7.0 & 11.9 & 11.6 \\
$\omega \mathrm{W}$ & 6.0 & 1.1 & 2.6 & 1.9 & 12.7 & 1.7 & 4.3 & 2.8 \\
\hline
\end{tabular}
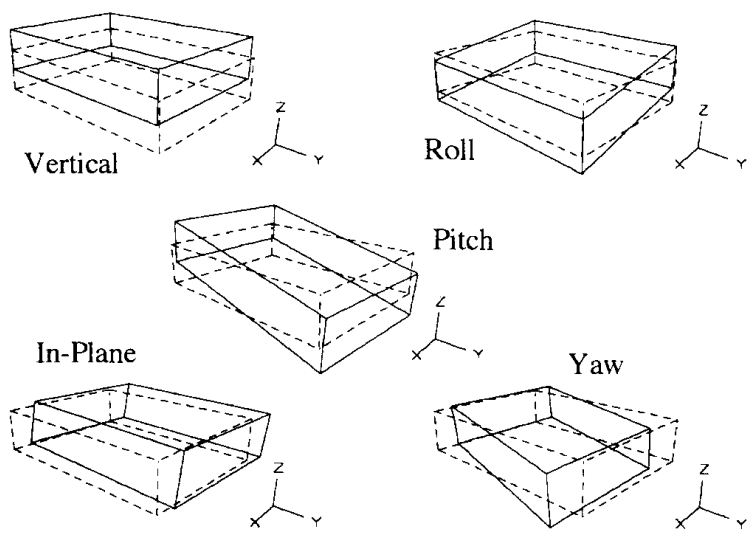

Fig. 19. Measured mode shapes of in-plane and out-of-plane motions of a five-turn silicon microgimbal.

in-plane mode corresponds to the side component of the roll motion; the second in-plane mode corresponds to the lateral bending of the serpentine microcoils (in-phase) and the third in-plane mode corresponds to the yaw motion (out-ofphase). The measurements are repeated at various locations of the slider/microgimbal to extrapolate the corresponding mode shapes of the measured resonances, which are shown in Fig. 19.

When operating in a disk drive, the roll motion of the microgimbal will be greatly suppressed due to the stiffness and inherent damping of the self-acting gas-lubricated slider bearings [4]. On the other hand, the in-plane and yaw modes will be unconstrained and will severely limit the bandwidth of the tracking servo system. Therefore, their frequencies need to be as high as possible (at least $>6 \mathrm{kHz}$, which is the fundamental reason for our desire to maintain a very high in-plane stiffness throughout the design process of the silicon microgimbals.

Table III summarizes our dynamic testing results for both the five-turn and the three-turn design (same as those presented in Table II). Recall that for a simple lumped-parameter model (where inertia can be assumed to be dominated by the slider and flexibility by the microgimbal), the resonant frequencies of the microgimbal can be estimated as follows:

$$
\omega_{\mathrm{wo}}=\sqrt{\frac{K_{z}}{M_{e}}} \text { and } \omega_{\mathrm{w}}=\sqrt{\frac{K_{z}}{M_{e}+M_{s}}}
$$

where $\omega_{\mathrm{w}}$ and $\omega_{\mathrm{wo}}$ are the measured resonant frequencies with and without bonded sliders, respectively, $K_{z}$ and $M_{e}$ are the equivalent spring constant and the effective mass of the microgimbal, respectively, and $M_{s}$ is the mass of the added slider.
TABLE IV

Comparison of Calculated Equivalent Inertia Properties of the Microgimbals and Measured Values of the Bonded Slider

\begin{tabular}{lccc}
\hline & 5-turn coil & 3-turn coil & slider \\
\cline { 2 - 4 } mass (mg) & 0.15 & 0.12 & 1.8 \\
pitch inertia $\left(\mathrm{mg}-\mathrm{mm}^{2}\right)$ & 0.064 & 0.050 & 0.34 \\
roll inertia $\left(\mathrm{mg}-\mathrm{mm}^{2}\right)$ & 0.031 & 0.016 & 0.26 \\
\hline
\end{tabular}
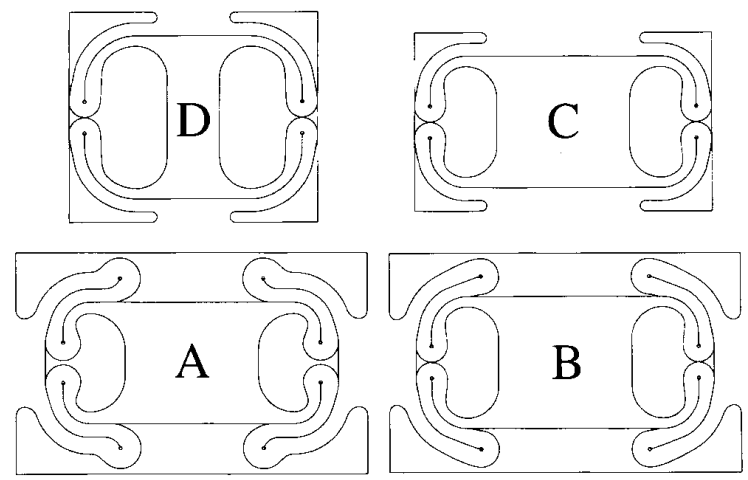

Fig. 20. Four different silicon microgimbals designs.

Therefore, for comparison purposes, we can calculate the equivalent spring constants of the microgimbals using the dynamic data tabulated in Table III. Such results have been summarized in Table II (bottom row) and one can see that the static and dynamic values are quite close, confirming the accuracy of our static measurements. Finally, Table IV compares the calculated equivalent mass and rotary inertia of the microgimbal with the measured values of the slider, which shows that the inertial properties of the microgimbal are about an order of magnitude lower than those of the slider, verifying the assumption of (2).

Our remaining testing efforts are concerned with a uniformity study of the fabrication process. Fig. 20 shows four different microgimbal designs: Design $\mathrm{A}$ is the three-turn design described earlier, Design B is a slightly modified three-turn design where we have increased the radius of the microcoils, Design $C$ is a two-turn design while Design D is a radically different design where the anchor points have been moved to the left- and right-hand sides of the center coupon. For each design, we fabricated a total of 30 samples (all with the improved fabrication process as described in Fig. 10, but half of the samples are coated with LTO instead of thermal oxide in order to investigate the effects, if any, of the hightemperature process on mechanical properties). On each of the samples, we performed dynamic testing to determine the spring stiffness. We found that the stiffness of the LTO samples is typically $5 \%$ higher than that of the thermal oxide samples (since the thickness of the LTO layer is about $2 \mu \mathrm{m}$ while that of the thermal oxide is less than $2000 \AA$, after TMAH). On the other hand, once the oxide layer is removed, the behaviors of the two sets of samples are basically the same. Fig. 21 shows the distribution of the measured vertical stiffness for Design A (with oxide removed). 


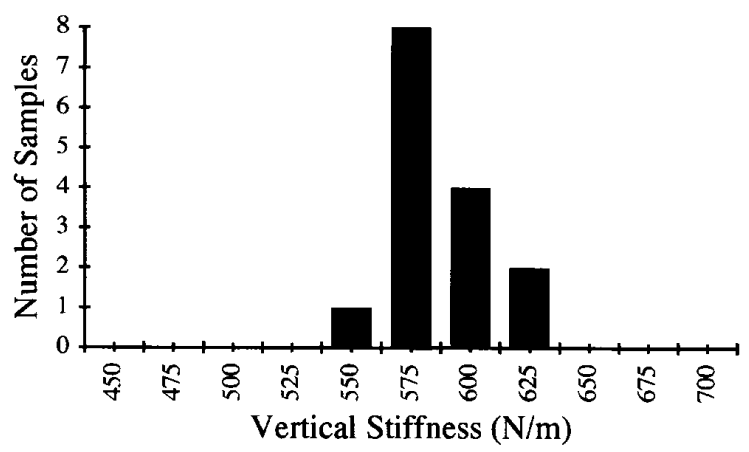

Fig. 21. Distribution of measured vertical stiffnesses of Design-A three-turn microgimbal.

TABLE V

Summary of Measured Vertical (N/m) and Pitch and Roll. StiffNesSES ( $\mu \mathrm{N}-\mathrm{m} / \mathrm{DEg}$ ) OF All Folr Microgimbal Designs

\begin{tabular}{ccccc}
\hline & $\mathrm{A}$ & $\mathrm{B}$ & $\mathrm{C}$ & $\mathrm{D}$ \\
\cline { 2 - 5 } vert & $576 \pm 4 \%$ & $524 \pm 2 \%$ & $704 \pm 3 \%$ & $404 \pm 6 \%$ \\
pitch & $12.8 \pm 4 \%$ & $12.3 \pm 1 \%$ & $15.0 \pm 6 \%$ & $5.5 \pm 2 \%$ \\
roll & $4.0 \pm 3 \%$ & $3.4 \pm 2 \%$ & $4.2 \pm 2 \%$ & $3.3 \pm 4 \%$ \\
\hline
\end{tabular}

Note that the average vertical stiffness value is quite a bit higher than that shown earlier in Table II (576 as opposed to $223 \mathrm{~N} / \mathrm{m}$ ). This is due to a thicker epi-layers (30 instead of $20 \mu \mathrm{m}$ ) and an improved RIE process (lower power, lower flow rate and lower process pressure) resulting in much smaller undercut, therefore wider beams (approximately $15 \mu \mathrm{m}$ wider) and higher spring stiffness.

We also find that uniformity in RIE is very important in terms of uniformity of the thickness of the finished silicon microgimbals. If RIE is not uniform, then once some of the microstructures are released, the plasma will start to etch the silicon from the backside causing damage to the silicon epilayers. In our process, we typically rotate the wafers two to three times in order to compensate for any variation in etch rate and sometimes even coat photoresist to the backside of the silicon microgimbal if necessary to increase protection.

From Fig. 21 one can see that the resulting variation is very small (less than $5 \%$ ) demonstrating that our fabrication process is indeed very reliable and uniform. Similar results are obtained for other samples and Table $\mathrm{V}$ summarizes the testing results of all four designs.

\section{CONCLUSION}

High-performance small form factor disk drives are becoming increasingly important for the emerging portable integrated computation/communication market. Silicon micromachining techniques offer many exciting opportunities for fabricating both passive microstructures and active electromagnetic microactuators for form factor reduction and recording density increase of future information storage products. In earlier papers, we have demonstrated a design concept for a silicon microgimbal/microsuspension which has certain performance advantages. In this latest paper, we document additional re- sults related to design optimization, process improvement and static/dynamic micron-scale component-level testing of a number of prototype designs.

In addition to computer disk drives, the results presented here have very wide applications, for example, in microrelays [2], microvalves [13], and many other micromachining applications requiring substantial out-of-plane motions [12].

\section{REFERENCES}

[1] S. S. Chiou and D. K. Miu, "Tracking dynamics of in-line suspensions in high-performance rigid disk drives with rotary actuators," ASME $J$. Vibration and Acoustics, vol. 114, pp. 67-73, Jan. 1992.

[2] H. Hosaka, H. Kuwano, and K. Yanagisawa, "Electromagnetic microrelays: concepts and fundamental characteristics," in Proc. IEEE MEMS-93 Workshop, 1993, pp. 12-17.

[3] W. P. Maszara and T. Thompson, "Strain compensation by $\mathrm{Ge}$ in B-doped silicon epitaxial films," J. Appl. Phys., vol. 72, no. 9, pp. 4477-4479, Nov. 1992.

[4] D. K. Miu and D. B. Bogy, "Dynamics of gas-lubricated slider bearings in magnetic recording disk files-Part I: Experimental observation," ASME J. Tribology, vol. 108, pp. 584-588, Oct. 1986.

[5] D. K. Miu and R. M. Karam, "Dynamics and design of read/write head suspensions for high-performance small form factor rigid disk drives," ASME Advances in Information Storage Systems, vol. 1, pp. 145-153, 1991.

[6] D. K. Miu and Y.-C. Tai, "Silicon Micromachined SCALED Technology," in Proc. 1993 JSME Int. Conf. Advanced Mechatronics, Tokyo, Japan, Aug. 2-4, 1993, pp. 527-532.

[7] D. K. Miu, S. Wu, S. Tatić-Lučić, and Y.-C. Tai, "Silicon micromachined microstructures for super-compact magnetic recording rigid disk drives," in Proc. 7th Int. Conf. Solid-State Sensors and Actuators, Yokohama, Japan, June 7-10, 1993, pp. 771-773.

[8] D. K. Miu, S. Wu, V. Temesvary, and Y.-C. Tai, "Silicon microgimbals for super-compact magnetic recording rigid disk drives," $A S M E$ Advances in Information Storage Systems, vol. 5, pp. 149-152, 1993.

[9] U. Schnakenberg, W. Benecke, B. Löchel, S. Ullerich, and P. Lange, " $\mathrm{NH}_{4} \mathrm{OH}$-based etchants for silicon micromachining: influence of additives and stability of passivation layers," Sensors and Actuators, vol. A-25-27, pp. 1-7, 1991.

[10] H. Seidel, L. Csepregi, A. Heuberger, and H. Baumgärtel, "Aniotropic etching of crystalline silicon in alkaline solutions-II, influence of dopants," J. Electrochem. Soc., vol. 137, no. 11, pp. 3626-3632, Nov. 1990.

[11] O. Tabata, R. Asahi, H. Funabashi, K. Shimaoka, and S. Sugiyama, "Anisotropic etching of silicon in TMAH solutions," Sensors and Actuators, vol. A-34, pp. 51-57, 1992.

[12] B. Wagner, W. Benecke, G. Engelmann, and J. Simon, "Microactuators with moving magnets for linear, torsional or multiaxial motion, Sensors and Actuators, vol. A-32, pp. 598-603, 1992.

[13] K. Yanagisawa, A. Tago, T. Ohkubo, and H. Kuwano, "Magnetic microactuator," in Proc. IEEE MEMS-91 Conf., 1991, pp. 120-124.

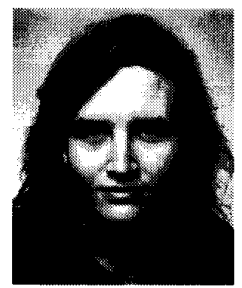

Viktoria Temesvary received the B.S. and M.S. degrees in mechanical engineering from UCLA with an emphasis in design and dynamics.

She has been with UCLA's Mechanical Engineering Department as a research associate since 1992. Her research interests include structural and magneto-static finite element simulation and design optimization of silicon-rnicromachined devices in rigid disk drive applications. 


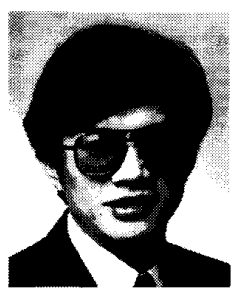

Shuyun $\mathrm{Wu}$ obtained the B.S. degree from the Department of Naval Architecture and Ocean Engineering in Shanghai Jiao Tong University in the People's Republic of China in 1987.

After graduation, he became a research engineer in the Information Storage Research Center where his work included head/disk dynamics, suspension design, and track accessing control. In 1992, he was invited to come to the United States as a visiting scholar to work with Professors Miu and Tai (Caltech) on silicon micromachining and its applications to computer disk drives. He is currently a graduate student in the Electrical Engineering Department at Caltech.

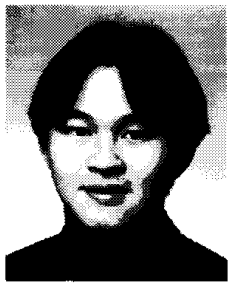

Wen H. Hsieh received his B.S. degree in electrical engineering from the California Institute of Technology in 1994 and is also currently a graduate student there. He has been working with Professors Tai and Miu on the application of silicon rnicromachining to computer disk drives since 1992

His research interests also include the study of thin film PTEE electrets and its application to silicon-micromachined devices.

Mr. Hsieh is a member of Tau Beta Pi, California Beta Chapter.

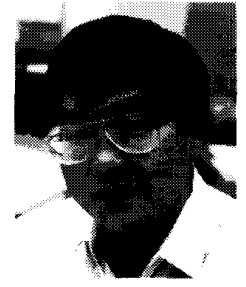

Yu-Chong Tai received his B.S. degree in electrical engineering from the National Taiwan University in 1981 and the M.S. and Ph.D. degrees from the University of California, Berkeley, in 1986 and 1989 , respectively.

After completing his education, he joined the faculty of Electrical Engineering at the California Institute of Technology, where he is currently an assistant professor. His research interests have included

silicon micromachining and its applications such as neural prosthetic devices, integrated millimeterwave waveguides, micro fluidic devices, miniature hard-disk drives, microphones, silicon hot-wire anemometers, magnetic milli and micro actuators, shock sensors, and MEMS systems for active fluid control. He is a member of the editorial board of the Journal of Micromechanics and Microengineering.

Dr. Tai received the Presidential Young Investigator Award and the David and Lucile Packard Fellowship in 1991

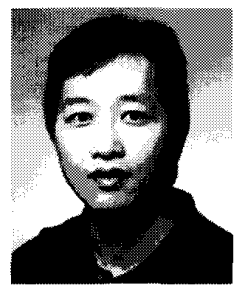

Denny K. Miu has been with the Mechanical Engineering Department at UCLA since 1987 and has been involved with the computer rigid disk drive industry since 1983.

His previous work include head/disk dynamics, suspension design, and residual vibrationless tracking accessing control. For the last three years, he has been working with Professor Yu-Chong Tai on silicon micromachining and its application to computer disk drives. He has published over 25 journal papers on mechanics and control issues related to computer disk drives as well as a graduate-level textbook with Springer-Verlag on advanced mechatronic problems of controlled electromechanical systems.

Dr. Miu is a recipient of the NSF Presidential Young Investigator Award and is also the founding Co-Chairman of the ASME Subdivision on Information Storage and Processing Systems. 